\title{
A ZERO THEOREM FOR THE TRANSFER OF COHERENT WITT GROUPS
}

\author{
STEFAN GILLE AND JENS HORNBOSTEL
}

\begin{abstract}
Let $R$ be a Gorenstein ring of finite Krull dimension and $t \in R$ a regular element. We show that if the quotient map $R \rightarrow R / R t$ has a flat splitting then the transfer morphism of coherent Witt groups $\operatorname{Tr}_{(R / R t) / R}: \tilde{W}^{i}(R / R t) \rightarrow \tilde{W}^{i+1}(R)$ is zero for all $i \in \mathbb{Z}$. As an application we give another proof of the Gersten conjecture for Witt groups in the case of regular semilocal rings essentially of finite type over a field of characteristic not 2.
\end{abstract}

\section{INTRODUCTION}

Let $\tilde{W}^{i}(R)$ be the $i$-th coherent Witt group of the Gorenstein ring of finite Krull dimension $R$ as defined in [11], and let $t \in R$ be a regular element, i.e. $t$ is not a zero divisor in $R$. Then $R / R t$ is a Gorenstein ring, too (see e.g. [8], Proposition 3.1.19), and we have a transfer morphism (cf. [11] and [12]):

$$
\operatorname{Tr}_{(R / R t) / R}: \tilde{W}^{i}(R / R t) \longrightarrow \tilde{W}^{i+1}(R)
$$

for all $i \in \mathbb{Z}$. The aim of this short note is to prove:

Theorem 0.1. Let $R$ be a Gorenstein ring of finite Krull dimension, $t \in R$ a regular element, and $\pi: R \longrightarrow R / R$ t the quotient map. Suppose $\pi$ has a flat splitting, i.e. there exists a flat morphism $q: R / R t \longrightarrow R$ such that $\pi \cdot q=\mathrm{id}_{R / R t}$. Then the transfer morphism

$$
\operatorname{Tr}_{(R / R t) / R}: \tilde{W}^{i}(R / R t) \longrightarrow \tilde{W}^{i+1}(R)
$$

is zero for all $i \in \mathbb{Z}$.

As special as this result sounds it has an interesting application, which we explain now. Balmer and Walter [5] have constructed a so-called augmented Gersten-Witt complex

$$
G W_{\bullet}(X): 0 \longrightarrow W(X) \longrightarrow \bigoplus_{x \in X^{(0)}} W(k(x)) \longrightarrow \bigoplus_{x \in X^{(1)}} W(k(x)) \longrightarrow \ldots
$$

for any regular scheme $X$ (here $X^{(i)} \subseteq X$ denotes the points of codimension $i$ and $k(x)$ the residue field at $x \in X)$. It is conjectured that this complex is exact if $X$ is the spectrum of a regular local ring $R$. This is known to be true if $\operatorname{dim} R<5$ by Balmer and Walter [5] and if $R$ contains a field by Balmer, Gille, Panin and Walter [4]. The proof in [4] uses a trick of Panin to go from the finite type case, proved by Balmer [3], to the general case. We give here another proof of Balmer's result, i.e. we show that $G W \bullet(X)$ is exact if $X$ is the spectrum of a semilocal regular ring of finite type over a field.

Our proof contains two new features. First, contrary to [3], we avoid the rather complicated variant of the normalization lemma from [9] (based on the work of Gabber [10]) and need only Quillen's normalization lemma. Second, Quillen uses additivity of the K-functor - which does

Date: January, 28, 2003. 
not hold for Witt groups - to conclude. We use the above zero theorem instead, which may hence be considered as a weak version of additivity for coherent Witt groups of Gorenstein rings.

\section{Terminology}

We assume that all rings are commutative, noetherian with 1 and have 2 invertible. Let $S$ be a ring. We denote $\mathcal{M}_{S}$ the category of all $S$-modules, $\mathcal{M}_{S}^{f g}$ the category of finitely generated $S$-modules and $\mathcal{P}_{S}$ the category of projective $S$-modules of finite rank. With $D^{b}(\mathcal{E})$ we denote the bounded derived category of the exact category $\mathcal{E}$, and $P_{\bullet} \longmapsto P_{\bullet}[1]=P_{\bullet}-1$ denotes the translation functor in $D^{b}(\mathcal{E})$. Let $D_{f g}^{b}\left(\mathcal{M}_{S}\right)$ be the full triangulated subcategory of $D^{b}\left(\mathcal{M}_{S}\right)$ consisting of complexes whose homology modules are in $\mathcal{M}_{S}^{f g}$. On this triangulated category $D_{f g}^{b}\left(\mathcal{M}_{S}\right)$ we have a codimension of support filtration:

$$
D_{f g}^{b}\left(\mathcal{M}_{S}\right)=D_{S}^{0} \supseteq D_{S}^{1} \supseteq \ldots \supseteq D_{S}^{n} \supseteq D_{S}^{n+1} \supseteq \ldots,
$$

where we have set

$$
D_{S}^{p}:=\left\{\begin{array}{l|l}
M_{\bullet} \in D_{f g}^{b}\left(\mathcal{M}_{S}\right) & \begin{array}{l}
H_{i}\left(M_{\bullet}\right)_{\wp}=0 \\
\text { and all } i \in \mathbb{Z} .
\end{array} \text { for all } \wp \in \operatorname{Spec} S \text { with } \operatorname{ht} \wp \leq p-1
\end{array}\right\}
$$

for $p \geq 0$. We use the following sign conventions for the total Hom and the tensor product of complexes. Let $P_{\bullet}, M_{\bullet}$, and $I_{\bullet}$ be bounded complexes of $S$-modules. Then $d^{P \otimes M}$ is given on $P_{r} \otimes_{S} M_{s}$ by $d_{r}^{P} \otimes \operatorname{id}_{M_{s}}+(-1)^{r} \operatorname{id}_{P_{r}} \otimes d_{s}^{M}$ and $d^{\operatorname{Hom}(M, I)}$ is defined on $\operatorname{Hom}_{S}\left(M_{-i-r}, I_{-r}\right)$ by $f \mapsto f d_{-i-r+1}^{M}+(-1)^{i+1} d_{-r}^{I} f$. We have then the following natural isomorphisms:

$$
\mathfrak{r}^{(i)}: P_{\bullet} \otimes\left(M_{\bullet}[i]\right) \stackrel{\simeq}{\longrightarrow}\left(P_{\bullet} \otimes M_{\bullet}\right)[i]
$$

given by $(-1)^{i j} \operatorname{id}_{P_{j} \otimes \ldots}$ on $P_{j} \otimes \ldots$, and

$$
\mathfrak{l}^{(i)}: P_{\bullet}[i] \otimes M_{\bullet} \stackrel{\simeq}{\rightarrow}\left(P_{\bullet} \otimes M_{\bullet}\right)[i]
$$

which is the identity in every degree.

If $S$ is a Gorenstein ring of finite Krull dimension and

$$
0 \longrightarrow S \longrightarrow I_{0} \longrightarrow I_{-1} \longrightarrow \ldots \longrightarrow I_{-\operatorname{dim} S} \longrightarrow 0
$$

a finite injective resolution of $S$, then $\chi_{f g}^{S}:=\operatorname{Hom}_{S}\left(-, I_{\bullet}\right)$ is a contravariant functor from $D_{f g}^{b}\left(\mathcal{M}_{S}\right)$ to itself, the so-called canonical duality. Here we consider $I_{\bullet}$ as an element of $D_{f g}^{b}\left(\mathcal{M}_{S}\right)$ with $I_{0}$ in degree 0 and $I_{-1}$ in degree $(-1)$. The category $D_{f g}^{b}\left(\mathcal{M}_{S}\right)$ equipped with this duality is a triangulated category with 1-exact duality in the sense of Balmer [1]. We denote the corresponding triangular Witt groups $\tilde{W}^{i}(S), i \in \mathbb{Z}$. These groups are called the coherent Witt groups of the ring $S$. They classify $i$-spaces in $D_{f g}^{b}\left(\mathcal{M}_{S}\right)$ for the canonical duality up to spaces having a Lagrangian. Recall that an $i$-space is a pair $\left[M_{\bullet}, \varphi\right]$ with $M_{\bullet} \in D_{f g}^{b}\left(\mathcal{M}_{S}\right)$ and $\varphi: M_{\bullet} \stackrel{\simeq}{\longrightarrow} \chi_{f g}^{S}\left(M_{\bullet}\right)[i]$ an isomorphism, such that $\chi_{f g}^{S}(\varphi) \cdot \varpi_{M}=\varphi$ where $\varpi: \operatorname{id}_{D_{f g}^{b}\left(\mathcal{M}_{S}\right)} \stackrel{\simeq}{\rightarrow} \chi_{f g}^{S} \chi_{f g}^{S}$ is the natural isomorphism to the bidual (cf. e.g. [11], Sect. 2.5.1). Observe that the full triangular subcategory $D_{S}^{p}$ of $D_{f g}^{b}\left(\mathcal{M}_{S}\right)$ is closed under the canonical duality and hence also a triangulated category with duality for all $p \geq 0$. We denote the corresponding triangular Witt groups by the symbols $W^{i}\left(D_{S}^{p}\right)(i \in \mathbb{Z}, p \geq 0)$.

Another example of a triangulated Witt groups which appears in this work are the derived Witt groups $W^{i}(S)$ of a ring $S$. These are by definition the triangular Witt groups of $D^{b}\left(\mathcal{P}_{S}\right)$ 
for the duality $\operatorname{Hom}_{S}(-, S)$. Recall that by a result of Balmer [1] the natural functor $\mathcal{P}_{S} \longrightarrow$ $D^{b}\left(\mathcal{P}_{S}\right)$ induces an isomorphism $W(S) \stackrel{\simeq}{\rightarrow} W^{0}(S)$ where $W(S)$ denotes the usual Witt group of the ring $S$.

For a regular ring $S$, we have an equivalence $D^{b}\left(\mathcal{P}_{S}\right) \stackrel{\simeq}{\longrightarrow} D_{f g}^{b}\left(\mathcal{M}_{S}\right)$ and thus an isomorphism $W^{i}(S) \cong \tilde{W}^{i}(S)$ for all $i \in \mathbb{Z}$. These isomorphisms are not natural for the following reason. To get a homomorphism between the Witt groups we have to make the natural embedding $F=F_{S}: D^{b}\left(\mathcal{P}_{S}\right) \hookrightarrow D_{f g}^{b}\left(\mathcal{M}_{S}\right)$ duality preserving, i.e. we need an isomorphism of functors $\eta: F \operatorname{Hom}_{S}(-, S) \stackrel{\simeq}{\longrightarrow} \operatorname{Hom}_{S}\left(F(-), I_{\bullet}\right)$ satisfying two compatibility axioms (cf. e.g. [11], Definition 2.6). These axioms make sure that $\left[F\left(P_{\bullet}\right), \eta_{P}[i] \cdot F(\varphi)\right]$ is an $i$-form in $D_{f g}^{b}\left(\mathcal{M}_{S}\right)$ if $\left[P_{\bullet}, \varphi\right]$ is an $i$-form in $D^{b}\left(\mathcal{P}_{S}\right)$ (cf. e.g. loc.cit. Theorem 2.7). Since $F(S)=S$ (considering $S$ as a complex which is concentrated in degree 0) the existence of such an isomorphism of functors $\eta$ is equivalent to the existence of a quasi isomorphism $\iota: S \stackrel{\simeq}{\longrightarrow} I_{\bullet}$. But there is no canonical choice for $\iota$, and therefore we have several isomorphisms $\eta$ making $F$ duality preserving which are all equally natural. Any such pair $(F, \eta)$ is called a duality preserving functor.

\section{Proof of Theorem 0.1}

We prove a more general result than announced in the introduction. Before we state it recall the following. The transfer $\operatorname{Tr}_{(R / R t) / R}$ is induced by the restriction of scalars functor $\pi_{*}: D_{f g}^{b}\left(\mathcal{M}_{R / R t}\right) \longrightarrow D_{f g}^{b}\left(\mathcal{M}_{R}\right)$. But $\pi_{*}\left(D_{R / R t}^{p}\right) \subseteq D_{R}^{p+1} \subseteq D_{R}^{p}$ and so we have also a transfer homomorphism $\operatorname{Tr}_{(R / R t) / R}: W^{i}\left(D_{R / R t}^{p}\right) \longrightarrow W^{i+1}\left(D_{R}^{p}\right)$.

Theorem 2.1. Let $R$ be a Gorenstein ring of finite Krull dimension, $t \in R$ a regular element, and $\pi: R \longrightarrow R / R$ t the quotient map. Suppose $\pi$ has a flat splitting, i.e. there exists a flat morphism $q: R / R t \longrightarrow R$ such that $\pi \cdot q=\mathrm{id}_{R / R t}$. Then the transfer morphism

$$
\operatorname{Tr}_{(R / R t) / R}: W^{i}\left(D_{R / R t}^{p}\right) \longrightarrow W^{i+1}\left(D_{R}^{p}\right)
$$

is zero for all $i \in \mathbb{Z}$ and $p \geq 0$.

Remark 2.2. Observe ([12], Remark 6.5) that $\operatorname{Tr}_{(R / R t) / R}: \tilde{W}^{i}(R / R t) \longrightarrow \tilde{W}^{i+1}(R)$ as defined in loc.cit. factors through the dévissage map $\tilde{W}^{i}(R / R t) \longrightarrow \tilde{W}_{R t}^{i+1}(R)$ described in [11], Sect. 4.1.

For the proof of Theorem 2.1 above we need

\subsection{Another description of coherent Witt groups.}

We recall some results from [11], Sect. 2.5. Let $S$ be a Gorenstein ring of finite Krull dimension and $j: S \longrightarrow J_{\bullet}$ a finite injective resolution of the $S$-module $S$. We denote $\mathcal{E}(S)$ the exact category of finitely generated $\operatorname{Hom}_{S}(-, S)$-acyclic $S$-modules. Since $S$ is Gorenstein this category has some useful properties. The contravariant functor $\operatorname{Hom}_{S}(-, S)$ is a duality on $\mathcal{E}(S)$ making it an exact category with duality (in particular all modules in $\mathcal{E}(S)$ are reflexive). We denote the corresponding derived Witt groups (cf. [2]) $W^{i}(\mathcal{E}(S)$ ). The natural functor $D^{b}(\mathcal{E}(S)) \longrightarrow D_{f g}^{b}\left(\mathcal{M}_{S}\right)$ is an equivalence $([6]$, Theorem 8.2). It becomes duality preserving using $\operatorname{Hom}_{S}(-, j)$ as duality transformation and we have ([11], Corollary 2.17) an isomorphism $F_{S, j}: W^{i}(\mathcal{E}(S)) \stackrel{\simeq}{\longrightarrow} \tilde{W}^{i}(S)$ for all $i \in \mathbb{Z}$. We want to point out that 
this isomorphism depends on $j$, a fact which we use below ( $c f$. end of last section). The triangulated category $D^{b}(\mathcal{E}(S))$ has also a filtration by full triangular subcategories:

$$
D^{b}(\mathcal{E}(S))=D^{b}(\mathcal{E}(S))^{(0)} \supseteq D^{b}(\mathcal{E}(S))^{(1)} \supseteq D^{b}(\mathcal{E}(S))^{(2)} \supseteq \ldots,
$$

where $D^{b}(\mathcal{E}(S))^{(p)}:=D^{b}(\mathcal{E}(S)) \cap D_{S}^{p}$. The restriction of $\operatorname{Hom}_{S}(-, S)$ is a duality on $D^{b}(\mathcal{E}(S))^{(p)}$ making this full subcategory a triangulated category with duality for all $p \geq 0$. We denote $W^{i}\left(D^{b}\left(\mathcal{E}(S)^{(p)}\right)\right.$ their triangular Witt groups.

If $S^{\prime}$ is another Gorenstein ring and $f: S \longrightarrow S^{\prime}$ is a flat morphism, we have a homomorphism of derived Witt groups $f^{*}: W^{i}(\mathcal{E}(S)) \longrightarrow W^{i}\left(\mathcal{E}\left(S^{\prime}\right)\right)$ for all $i \in \mathbb{Z}$ which is induced by the exact functor $M_{\bullet} \longmapsto S^{\prime} \otimes_{S} M_{\bullet}$.

We have a pairing $W^{d}(S) \times W^{m}(\mathcal{E}(S)) \longrightarrow W^{d+m}(\mathcal{E}(S))$. It is defined by considering $P_{\bullet} \in D^{b}\left(\mathcal{P}_{S}\right)$ as duality preserving functor, made duality preserving using a form on it. We explain this briefly and refer to [13] for details and more information. Let $x=\left[P_{\bullet}, \varphi\right] \in W^{d}(S)$. Then we have the following isomorphism $\eta_{M}^{x}$ :

$$
\begin{aligned}
P \bullet & \otimes_{S} \operatorname{Hom}_{S}\left(M_{\bullet}, S\right) \stackrel{\varphi \otimes \mathrm{id}}{\longrightarrow} \operatorname{Hom}_{S}\left(P_{\bullet}, S\right)[d] \otimes_{S} \operatorname{Hom}_{S}\left(M_{\bullet}, S\right) \\
& \stackrel{\mathfrak{r}^{(d)}}{\longrightarrow}\left(\operatorname{Hom}_{S}\left(P_{\bullet}, S\right) \otimes_{S} \operatorname{Hom}_{S}\left(M_{\bullet}, S\right)\right)[d] \simeq \operatorname{Hom}_{S}\left(P_{\bullet} \otimes_{S} M_{\bullet}\right)[d]
\end{aligned}
$$

natural in $M_{\bullet} \in D^{b}(\mathcal{E}(S))$. It is a duality transformation for the exact functor $P_{\bullet} \otimes_{S}-$, and so we get a homomorphism $x \star_{l}-: W^{m}(\mathcal{E}(S)) \longrightarrow W^{d+m}(\mathcal{E}(S))$, the so-called left product with $x$. It sends the $m$-space $\left[M_{\bullet}, \psi\right] \in W^{s}(\mathcal{E}(S))$ to the following $(d+m)$-space:

$$
P_{\bullet} \otimes_{S} M_{\bullet} \stackrel{\operatorname{id} \otimes \psi}{\longrightarrow} P \bullet \otimes_{S}\left(\operatorname{Hom}_{S}\left(M_{\bullet}, S\right)[m]\right) \stackrel{(-1)^{d m} \eta_{M}^{x}[m] \cdot \mathfrak{r}^{(m)}}{\longrightarrow} \operatorname{Hom}_{S}\left(P_{\bullet} \otimes_{S} M_{\bullet}, S\right)[d+m]
$$

(cf. [13], Remark 1.9, Theorem 2.6, and Theorem 2.9).

After these preparations we can start with the proof of Theorem 2.1.

\subsection{A homomorphism $W^{i}(\mathcal{E}(R / R t)) \longrightarrow W^{i+1}(\mathcal{E}(R))$.}

Denote $K(t)$ the (homological) Koszul complex of $t$. We consider $K(t)$ as a complex in $D^{b}\left(\mathcal{P}_{R}\right)$ living in degrees 1 and 0 . On it there is the "standard" 1 -form $\ell_{1}$ :

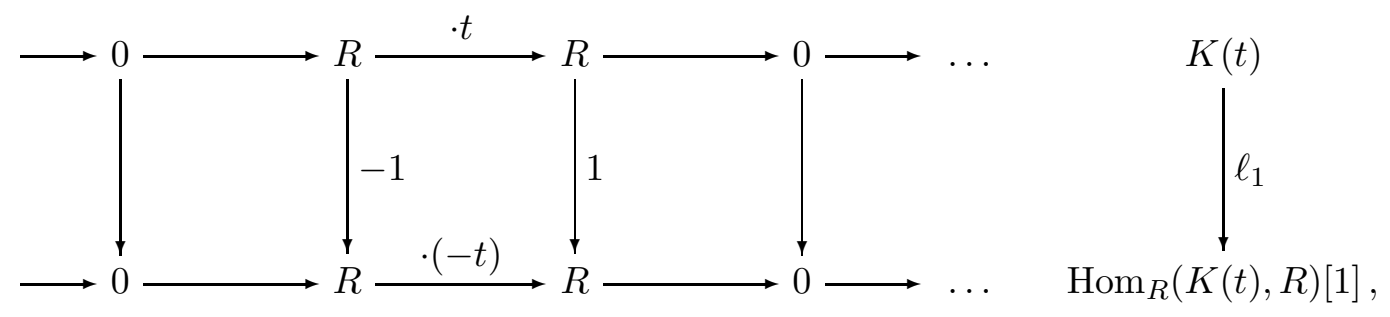

$\operatorname{deg} 1 \quad \operatorname{deg} 0$

where we have identified $R=\operatorname{Hom}_{R}(R, R)$. This space $\left[K(t), \ell_{1}\right]$ is zero in the derived Witt group $W^{1}(R)$ because
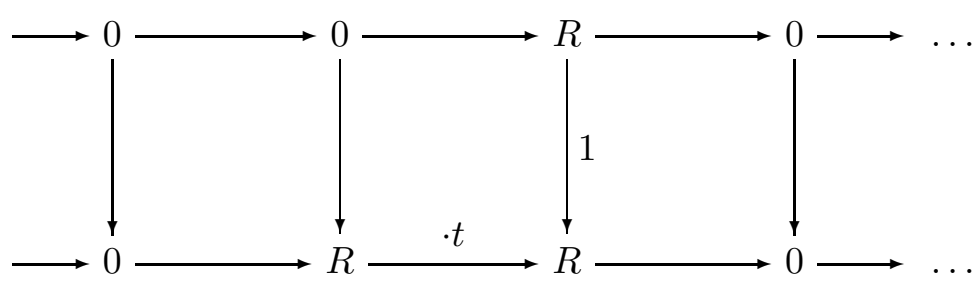
is a Lagrangian. Therefore the following homomorphism

$$
W^{i}(\mathcal{E}(R / R t)) \longrightarrow W^{i+1}(\mathcal{E}(R)) \quad\left[M_{\bullet}, \varphi\right] \longmapsto\left[K(t), \ell_{1}\right] \star_{l} q^{*}\left(\left[M_{\bullet}, \varphi\right]\right)
$$

is zero. Now a space is zero in a triangular Witt group if and only if it has a Lagrangian ([1], Theorem 3.5). We now give a Lagrangian of $\left[K(t), \ell_{1}\right] \star_{l} q^{*}\left(\left[M_{\bullet}, \varphi\right]\right)$. By [13], Lemma 1.15 there is an isometry $\left[K(t), \ell_{1}\right] \star_{l} q^{*}\left(\left[M_{\bullet}, \varphi\right]\right) \simeq(-1)^{i}\left[K(t), \ell_{1}\right] \star_{r} q^{*}\left(\left[M_{\bullet}, \varphi\right]\right)$, where $\star_{r}$ is the right product. This right product is defined analogous to the left product: We make the exact functor $-\otimes q^{*}\left(M_{\bullet}\right): D^{b}\left(\mathcal{P}_{R}\right) \longrightarrow D^{b}(\mathcal{E}(R))$ duality preserving using the form induced by $\varphi$ on $q^{*}\left(M_{\bullet}\right)$ ([13], Sect. 1.3). From the theory of duality preserving functors it follows that $R \otimes_{R} q^{*}\left(M_{\bullet}\right)$ is a Lagrangian of $(-1)^{i}\left[K(t), \ell_{1}\right] \star_{r} q^{*}\left(\left[M_{\bullet}, \varphi\right]\right)$ and so of $\left[K(t), \ell_{1}\right] \star_{l} q^{*}\left(\left[M_{\bullet}, \varphi\right]\right)$ as well, since $R$ (considered as a complex concentrated in degree 0 ) is a Lagrangian of $K(t)$. Note that due to the flatness of $q$ we have $q^{*}\left(D^{b}(\mathcal{E}(R / R t))^{(p)}\right) \subseteq D^{b}(\mathcal{E}(R))^{(p)}$. It follows that $\left[K(t), \ell_{1}\right] \star_{l} q^{*}\left(\left[M_{\bullet}, \varphi\right]\right)$ is not only zero in $W^{i+1}(\mathcal{E}(R))$, but also in $W^{i+1}\left(D^{b}\left(\mathcal{E}(R)^{(p)}\right)\right)$ if $M_{\bullet} \in D^{b}\left(\mathcal{E}(R / R t)^{(p)}\right)$. We state this as a Lemma.

Lemma 2.5. Let $\left[M_{\bullet}, \varphi\right]$ be an $i$-space with $M_{\bullet} \in D^{b}(\mathcal{E}(R / R t))^{(p)}$ for some $p \geq 0$. Then the space

$$
\left[K(t), \ell_{1}\right] \star_{l} q^{*}\left(\left[M_{\bullet}, \varphi\right]\right)
$$

is zero in $W^{i+1}\left(D^{b}(\mathcal{E}(R))^{(p)}\right)$.

\subsection{Compatible injective resolutions.}

We want to choose injective resolutions $\kappa: R \rightarrow I_{\bullet}$ and $\epsilon: R / R t \rightarrow E_{\bullet}$ which are compatible in a sense which is explained by Lemma 2.8 below. Let for this

$$
0 \longrightarrow R \stackrel{\kappa}{\longrightarrow} I_{0} \stackrel{d_{0}^{I}}{\longrightarrow} I_{-1} \stackrel{d_{-1}^{I}}{\longrightarrow} \ldots \stackrel{d_{-(n-1)}^{I}}{\longrightarrow} I_{-n} \longrightarrow 0 \quad n=\operatorname{dim} R
$$

be a minimal injective resolution of $R$ (cf. [8], Sect. 3.2), and

$$
E_{-r}:=I_{-(r+1)}^{t}:=\left\{h \in I_{-(r+1)} \mid t \cdot h=0\right\} \quad \text { and } \quad d_{-r}^{E}:=\left.d_{-(r+1)}^{I}\right|_{I_{-(r+1)}^{t}} .
$$

It is shown in [11], Sect. 3.1, in particular Theorem 3.5, that $E$ • is a minimal injective resolution of $\operatorname{ker} d_{0}^{E} \simeq R / R t$. We need a particular isomorphism $R / R t \stackrel{\simeq}{\longrightarrow} \operatorname{ker} d_{0}^{E}$.

Lemma 2.7. $\quad$ a) Multiplication by $t: I_{0} \longrightarrow I_{0}, y \longmapsto t \cdot y$ is an isomorphism. We denote the inverse $t^{-1}$.

b) The kernel of the composition

$$
R \stackrel{\kappa}{\longrightarrow} I_{0} \stackrel{t^{-1}}{\longrightarrow} I_{0} \stackrel{d_{0}^{I}}{\longrightarrow} I_{-1}
$$

is the ideal Rt and the image is ker $d_{0}^{E}$. In particular we have an isomorphism

$$
\epsilon: R / R t \stackrel{\simeq}{\longrightarrow} \operatorname{ker} d_{0}^{E} \quad \pi(r) \longmapsto d_{0}^{I}\left(t^{-1} \kappa(r)\right) .
$$

Proof. a) By definition $R \stackrel{\kappa}{\rightarrow} I_{0}$ is an injective hull of $R$ and so in particular an essential extension. It follows that $\operatorname{ker}\left(I_{0} \stackrel{\cdot t}{\rightarrow} I_{0}\right) \neq 0$ if and only if $\operatorname{ker}(R \stackrel{\cdot t}{\rightarrow} R) \neq 0$. But $t$ is not a zero divisor and so multiplication by $t$ is an injection. We get a decomposition $I_{0} \simeq t I_{0} \oplus H$ for some submodule $H \subset I_{0}$ because $I_{0}$ is injective. But this is only possible for $H=0$ since over a commutative noetherian ring any injective module has a unique decomposition into indecomposable injective modules (cf. e.g. [8], Proposition 3.2.8). 
b) It is obvious that the kernel of this composition is the ideal $R t$ and that the image lies in $\operatorname{ker} d_{0}^{E}$. Let $e \in \operatorname{ker} d_{0}^{E} \subseteq \operatorname{ker} d_{-1}^{I}$. Then there is an $e^{\prime} \in I_{0}$, such that $d_{0}^{I}\left(e^{\prime}\right)=e$. Since $t \cdot e=0$ we have $t \cdot e^{\prime} \in \operatorname{ker} d_{0}^{I}=\operatorname{Im} \kappa$, and so $t \cdot e^{\prime}=\kappa(r)$ for some $r \in R$.

Therefore

$$
0 \longrightarrow R / R t \stackrel{\epsilon}{\longrightarrow} E_{0} \stackrel{d_{0}^{E}}{\longrightarrow} E_{-1} \longrightarrow \ldots \stackrel{d_{-(n-1)}^{E}}{\longrightarrow} E_{-(n-1)} \longrightarrow 0
$$

is a minimal injective resolution. Theorem 2.1 now follows from Lemma 2.5 and the following surprising

Lemma 2.8. Let $x$ be an $i$-space representing an element of $W^{i}(\mathcal{E}(R / R t))$. Then there is an isometry

$$
F_{R, \kappa}\left(\left[K(t), \ell_{1}\right] \star_{l} q^{*}(x)\right) \stackrel{\simeq}{\longrightarrow} \operatorname{Tr}_{(R / R t) / R}\left(F_{R / R t, \epsilon}(x)\right) .
$$

Remark 2.9. If $R / R t$ is regular one can give a quite simple proof of this lemma using the projection formula [12], Theorem 5.2 (cf. the proof of loc.cit. Theorem 9.3).

Proof. For brevity we will use the following notation. Let $M \in \mathcal{M}_{R / R t}$ and $N \in \mathcal{M}_{R}$. We set

$$
M^{R}:=R \otimes_{R / R t} M \quad \text { and } \quad D_{i}(N):=\operatorname{Hom}_{R}\left(N, I_{-i}\right) .
$$

Let $x=\left[M_{\bullet}, \varphi\right]$. We may assume that $\varphi$ is a morphism of complexes and not a fraction. This is no restriction: If $\varphi$ is given by a fraction $M_{\bullet} \stackrel{\alpha}{\leftarrow} H_{\bullet} \stackrel{\beta}{\rightarrow} \operatorname{Hom}_{R / R t}\left(M_{\bullet}, R / R t\right)[i]$, then $\left[H_{\bullet}, \operatorname{Hom}_{R / R t}(\alpha, R / R t)[i] \cdot \beta\right]$ is an $i$-space in $D^{b}(\mathcal{E}(R / R t))$ which is isometric to $\left[M_{\bullet}, \varphi\right]$ via the isomorphism $\alpha: H_{\bullet} \stackrel{\simeq}{\rightarrow} M_{\bullet}$. Hence assume $\varphi$ is given in degree $l$ by the following $R / R t$-linear map:

$$
\varphi_{l}: M_{l} \longrightarrow \operatorname{Hom}_{R / R t}\left(M_{-l+i}, R / R t\right) .
$$

Then $q^{*}(x)$ is given in degree $l$ by $\varphi_{l}^{R}: M_{l}^{R} \longrightarrow \operatorname{Hom}_{R}\left(M_{-l+i}^{R}, R\right)$ which sends the element $r \otimes m \in M_{l}^{R}=R \otimes_{R / R t} M_{l}$ to the following $R$-linear map

$$
M_{-l+i}^{R} \longrightarrow R \quad r^{\prime} \otimes m^{\prime} \longmapsto\left(r r^{\prime}\right) \cdot q\left(\varphi_{l}(m)\left(m^{\prime}\right)\right) .
$$

It follows that $F_{R, \kappa}\left(\left[K(t), \ell_{1}\right] \star_{l} q^{*}(x)\right)$ is given in degree $l$ by (cf. the discussion above):

$$
\begin{gathered}
\Theta_{l}:=\left(\begin{array}{cc}
0 & -\kappa \varphi_{l-1}^{R} \\
(-1)^{i} \kappa \varphi_{l}^{R} & 0 \\
0 & 0 \\
\vdots & \vdots \\
0 & 0
\end{array}\right) \quad: \\
M_{l}^{R} \oplus M_{l-1}^{R} \longrightarrow\left(D_{0} M_{-l+i+1}^{R} \oplus D_{0} M_{-l+i}^{R}\right) \oplus\left(D_{1} M_{-l+i}^{R} \oplus D_{1} M_{-l+i-1}^{R}\right) \oplus \ldots .
\end{gathered}
$$

The space $\operatorname{Tr}_{(R / R t) / R}\left(F_{R / R t, \epsilon}\left(\left[M_{\bullet}, \varphi\right]\right)\right)$ on the complex of $R$-modules $M_{\bullet}$ is given in degree $l$ by (cf. [11], proof of Theorem 4.2; note that we get a $(-1)^{i}$ because we use the $i$-th shift of 
the duality transformation, cf. loc.cit. proof of Theorem 2.7):

$$
\Xi_{l}:=\left(\begin{array}{c}
0 \\
(-1)^{l} \epsilon \varphi_{l} \\
0 \\
\vdots \\
0
\end{array}\right) \quad:
$$

$$
M_{l} \quad \longrightarrow \quad D_{0} M_{-l+i+1} \oplus D_{1} M_{-l+i} \oplus D_{2} M_{-l+i-1} \oplus \ldots
$$

Because the morphism of rings $q$ is flat the following sequence of complexes of $R$-modules

$$
0 \longrightarrow M_{\bullet}^{R} \stackrel{t \otimes \mathrm{id}}{\longrightarrow} M_{\bullet}^{R} \stackrel{r \otimes m \mapsto \pi(r) \cdot m}{\longrightarrow} M_{\bullet} \longrightarrow 0
$$

is exact for any $M_{\bullet} \in D_{f g}^{b}\left(\mathcal{M}_{R / R t}\right)$. The tensor product $K(t) \otimes_{R} M_{\bullet}^{R}$ is isomorphic to the total complex of $M_{\bullet}^{R} \stackrel{t \otimes \text { id }}{\longrightarrow} M_{\bullet}^{R}$. Hence the exact sequence of complexes above yields a quasi-isomorphism of complexes of $R$-modules $\alpha: K(t) \otimes_{R} M_{\bullet}^{R} \longrightarrow M_{\bullet}$ given in degree $l$ by

$$
\left(R \otimes_{R / R t} M_{l}\right) \oplus\left(R \otimes_{R / R t} M_{l-1}\right) \longrightarrow M_{l} \quad\left(r \otimes m, r^{\prime} \otimes m^{\prime}\right) \longmapsto \pi(r) \cdot m .
$$

We claim that $\alpha$ is the desired isometry. This follows from the following fact whose straightforward proof is left to the reader (use Lemma 2.7 and the sign conventions of section 1). The morphisms

$$
\begin{gathered}
s_{l}:=\left(\begin{array}{ccc}
t^{-1} \kappa \varphi_{l}^{R} & 0 \\
0 & 0 \\
0 & 0 \\
\vdots & \vdots \\
0 & 0
\end{array}\right) \quad: \\
M_{l}^{R} \oplus M_{l-1}^{R} \quad \longrightarrow \quad\left(D_{0} M_{-l+i}^{R} \oplus D_{0} M_{-l+i-1}^{R}\right) \oplus\left(D_{1} M_{-l+i-1}^{R} \oplus D_{1} M_{-l+i-2}^{R}\right) \oplus \ldots .
\end{gathered}
$$

define a homotopy between $\Theta$ and $\operatorname{Hom}_{R}\left(\alpha, I_{\bullet}\right)[i+1] \cdot \Xi \cdot \alpha$.

\section{The proof of the Gersten conjecture for Witt groups via Quillen NORMALIZATION}

\subsection{The spectral sequence.}

We now recall the definition of the Gersten-Witt spectral sequence. More details and proofs can be found in [5] (regular schemes) and [11] (Gorenstein schemes of finite Krull dimension). Let $S$ be a Gorenstein ring with $\operatorname{dim} S=n<\infty$, and $I_{\bullet}$ a finite injective resolution of $S$. The canonical duality $\operatorname{Hom}_{S}\left(-, I_{\bullet}\right)$ induces a duality on the full triangulated category $D_{S}^{p}$ and so we have an exact sequence of triangulated categories with duality

$$
0 \longrightarrow D_{S}^{p+1} \longrightarrow D_{S}^{p} \longrightarrow D_{S}^{p} / D_{S}^{p+1} \longrightarrow 0
$$


for all $p \geq 0$. Applying Balmer's [1] localization theorem, we get a long exact sequence of triangular Witt groups

$$
\ldots \longrightarrow W^{i}\left(D_{S}^{p+1}\right) \longrightarrow W^{i}\left(D_{S}^{p}\right) \longrightarrow W^{i}\left(D_{S}^{p} / D_{S}^{p+1}\right) \stackrel{\partial}{\longrightarrow} W^{i+1}\left(D_{S}^{p+1}\right)
$$

for all $p \geq 0$ and $i \in \mathbb{Z}$, and so (using Massey's method of exact couples) a convergent spectral sequence, the so-called Gersten Witt spectral sequence:

$$
E_{1}^{p, q}(S):=W^{p+q}\left(D_{S}^{p}\right) \quad \Longrightarrow \quad \tilde{W}^{p+q}(S) .
$$

It follows from the dévissage result [11], Corollary 3.11 that $E_{1}^{p, q}(S)=0$ if $q \not \equiv 0 \bmod 4$ and that there is a non-canonical isomorphism $E_{1}^{p, 4 s}(S) \simeq \bigoplus_{\text {ht } \wp=p} W(k(\wp))$ for all $s \in \mathbb{Z}$, where $k(\wp)$ is the residue field at $\wp \in \operatorname{Spec} S$. The zero line of this spectral sequence composed with the natural homomorphism $W(S)=W^{0}\left(D_{S}^{0}\right) \longrightarrow W^{0}\left(D_{S}^{0} / D_{S}^{1}\right)$ is the augmented Gersten Witt complex $G W_{\bullet}(S)$ :

$$
0 \longrightarrow W(S) \longrightarrow \bigoplus_{\mathrm{ht} \wp=0} W(k(\wp)) \longrightarrow \bigoplus_{\mathrm{ht}}^{\wp=1} W W(k(\wp)) \longrightarrow \ldots
$$

\subsection{The Gersten conjecture for Witt groups.}

This conjecture asserts that $G W_{\bullet}(A)$ is exact for any regular local ring $A$. Our aim here is to give another proof of this conjecture for regular semilocal rings of finite type over a field.

Theorem 3.3. Let $A$ be a regular semilocal ring essentially of finite type over a field $k$. Then the augmented Gersten-Witt complex $G W_{\bullet}(A)$ :

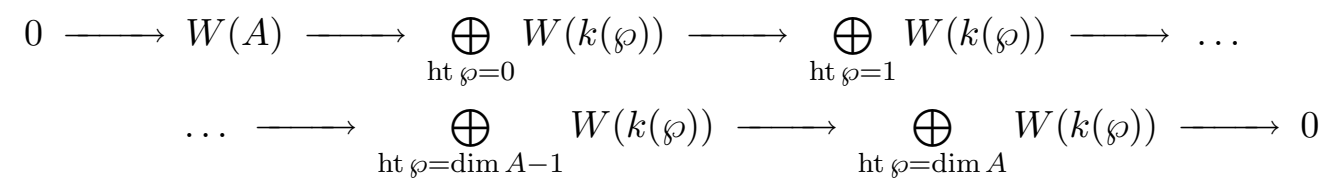

is exact.

Remark 3.4. As already mentioned in the introduction this has been proved before. Namely, Balmer [3] has proved this if the field is infinite (of course one easily deduces from this the case of a finite field, cf. [4], Sect. 5). Since we can use Quillen's [15] variant of the normalization lemma our proof works at once for any field.

Proof. By well known arguments (e.g. [15], Proposition 5.6) it is enough to show that the natural homomorphism $W^{i}\left(D_{A}^{p+1}\right) \longrightarrow W^{i}\left(D_{A}^{p}\right)$ is zero for all $p \geq 0$ and all $i \in \mathbb{Z}$. Let $x \in W^{i}\left(D_{A}^{p+1}\right)$. Since $A$ is essentially of finite type over the field $k$, there exists a finitely generated $k$-algebra $R$ which is smooth at a finite set of points $S$ such that $A=U^{-1} R$, where $U=R \backslash \bigcup_{\wp \in S} \wp$. Hence there exists $g \notin \bigcup_{\wp \in S} \wp$ such that $x$ is in the image of $W^{i}\left(D_{R_{g}}^{p+1}\right) \longrightarrow$ $W^{i}\left(D_{A}^{p+1}\right)$. Replacing $R$ by $R_{g^{\prime}}$ for some $g^{\prime} \notin \bigcup_{\wp \in S} \wp$ we may assume that $R$ is smooth over $k$ and that $x \in \operatorname{Im}\left(W^{i}\left(D_{R}^{p+1}\right) \longrightarrow W^{i}\left(D_{A}^{p+1}\right)\right)$. Hence the theorem follows from

Lemma 3.5. Let $R$ be an algebra essentially of finite type over a field $k$ which is smooth over $k$ and $S \subseteq \operatorname{Spec} R$ a finite set. Then for any $x \in W^{i}\left(D_{R}^{p+1}\right)$ there exists an element $f \notin \bigcup_{\wp \in S} \wp$, such that the homomorphism

$$
W^{i}\left(D_{R}^{p+1}\right) \longrightarrow W^{i}\left(D_{R_{f}}^{p}\right)
$$

is zero. 
Proof. Let $x=\left[M_{\bullet}, \phi\right] \in W^{i}\left(D_{R}^{p+1}\right)$. Then by definition $\operatorname{codim}\left(\operatorname{supp} M_{\bullet}\right) \geq p+1 \geq 1$, where

$$
\operatorname{supp} M_{\bullet}:=\left\{\wp \in \operatorname{Spec} R \mid H_{i}\left(M_{\bullet}\right)_{\wp} \neq 0 \quad \text { for some } i \in \mathbb{Z}\right\} \text {. }
$$

It follows from dévissage [11], Theorem 4.1 that there exists a regular $t \in R$, such that

$$
x \in \operatorname{Im}\left(\operatorname{Tr}_{(R / R t) / R}: W^{i-1}\left(D_{R / R t}^{p}\right) \longrightarrow W^{i}\left(D_{R}^{p}\right)\right)
$$

Therefore it is enough to show that the composition

$$
W^{i-1}\left(D_{R / R t}^{p}\right) \stackrel{\operatorname{Tr}_{(R / R t) / R}}{\longrightarrow} W^{i}\left(D_{R}^{p}\right) \longrightarrow W^{i}\left(D_{R_{f}}^{p}\right)
$$

is zero for some $f \notin \bigcup_{\wp \in S} \wp$. Let $\rho$ be the quotient map $R \longrightarrow R / R t$. We now use the following normalization lemma of Quillen ([15], §7, Lemma 5.12 and the discussion thereafter).

Lemma 3.6. There exists an $f \notin \bigcup_{\wp \in S} \wp$ and a k-algebra $C$, such that there is

a) a finite morphism $v: R_{f} \longrightarrow C$,

b) a surjective map $\pi: C \longrightarrow(R / R t)_{f}$, such that

i) $\operatorname{ker} \pi=C \cdot t^{\prime}$ with $t^{\prime}$ regular, and

ii) the following diagram commutes

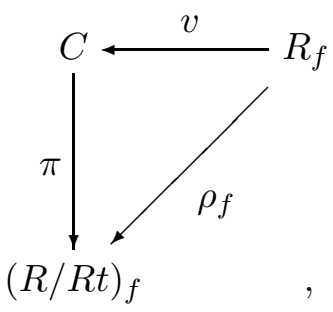

and

c) a smooth morphism $q:(R / R t)_{f} \longrightarrow C$ essentially of finite type which splits $\pi$, i.e. $\pi \cdot q=\operatorname{id}_{(R / R t)_{f}}$.

Since $t$ is regular the ring $R / R t$ is Gorenstein. Now $q:(R / R t)_{f} \longrightarrow C$ is smooth and so in particular flat. Moreover since smoothness is preserved by base change we know that all fibers of $q$ are smooth over the appropriate residue fields (cf. e.g. [7], Proposition 2.4/8). It follows that $q$ has regular fibers (cf. e.g. [7], Theorem 2.2/15) and hence $C$ is Gorenstein by [8], Corollary 3.3.15. Therefore we can apply Theorem 2.1 to see that

$$
\operatorname{Tr}_{(R / R t)_{f} / C}: W^{i-1}\left(D_{(R / R t)_{f}}^{p}\right) \longrightarrow W^{i}\left(D_{C}^{p}\right)
$$

is the zero map and hence $\operatorname{Tr}_{(R / R t)_{f} / R_{f}}: W^{i-1}\left(D_{(R / R t)_{f}}^{p}\right) \longrightarrow W^{i}\left(D_{R_{f}}^{p}\right)$, too, since we have $\operatorname{Tr}_{(R / R t)_{f} / R_{f}}=\operatorname{Tr}_{C / R_{f}} \cdot \operatorname{Tr}_{(R / R t)_{f} / C}$ by elementary properties of the transfer for coherent Witt groups stated in [12], Sect. 4. From this reference we also know that the following diagram 
commutes:

and so we are done.

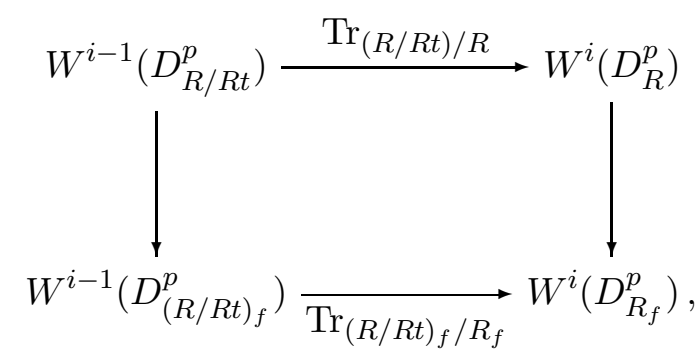

Remark 3.7. Note that this gives also a new proof of an old result of Ojanguren [14] which asserts that for any regular local ring $A$ essentially of finite type over a field $k$ and with quotient field $K$ the natural morphism $W(A) \longrightarrow W(K)$ is injective.

\section{REFERENCES}

[1] P. Balmer, Triangular Witt groups. Part I: The 12-term localization exact sequence, K-Theory 19 (2000), $311-363$

[2] P. Balmer, Triangular Witt groups. Part II: From usual to derived, Math. Z. 236 (2001), 351-382

[3] P. Balmer, Witt cohomology, Mayer-Vietoris, homotopy invariance, and the Gersten conjecture, K-Theory 23 (2001), 15-30.

[4] P. Balmer, S. Gille, I. Panin, C. Walter, The Gersten conjecture for Witt groups in the equicharacteristic case, Documenta Math. 7 (2002), 203-217

[5] P. Balmer, C. Walter, A Gersten-Witt spectral sequence for regular schemes, Ann. Scient. Éc. Norm. Sup. (4) 35 (2002), 127-152.

[6] H. Bass, The ubiquity of Gorenstein rings, Math. Z. 82 (1963), 8-28

[7] S. Bosch, W. Lütkebohmert, M. Raynaud, Néron models, Springer 1990

[8] W. Bruns, J. Herzog, Cohen-Macaulay rings, Cambridge Univ. Press, 1993

[9] J.-L. Colliot-Thélène, R.T. Hoobler, B. Kahn, The Bloch-Ogus-Gabber Theorem, Algebraic K-theory (Toronto, 1996), 31-94, Fields Inst. Commun., 16, Amer. Math. Soc., Providence, 1997.

[10] O. Gabber, Gersten's conjecture for some complexes of vanishing cycles, Manuscripta Math. 85 (1994), 323-343

[11] S. Gille, On Witt groups with support, Math. Ann. 322 (2002), 103-137

[12] S. Gille, A transfer morphism for Witt groups, to appear J. reine angew. Math.

[13] S. Gille, A. Nenashev, Pairings in triangular Witt theory, J. Algebra 261 (2003), 292-309

[14] M. Ojanguren, Quadratic forms over regular rings, J. Indian Math. Soc. (N.S.) 44 (1980), 109-116.

[15] D. Quillen, Higher algebraic K-theory, Algebraic K-theory I: Higher K-theories, (Proc. Conf., Battele Memorial Inst., Seattle, Wash., 1972), 85-147, LNM 341, Springer 1973

Stefan Gille, SFB 478, Universität Münster, Hittorfstrasse 27, 48149 Münster, Germany

E-mail address: gilles@math.uni-muenster.de

Jens Hornbostel, Universität Regensburg, NWF I - Mathematik, 93040 Regensburg

E-mail address: jens.hornbostel@mathematik.uni-regensburg.de 